\title{
CHALLENGES, STRATEGIES AND TECHNIQUES FOR INTERNATIONAL TRAINING IN TECHNOLOGY FOR CULTURAL HERITAGE CONSERVATION
}

\author{
R. Eppich ${ }^{\mathrm{a},}$ A. Almagro Vidal ${ }^{\mathrm{b}}$ \\ a Tecnalia Research \& Innovation, Parque Tecnológico de Vizcaya, Derio, Spain. rand.eppich@tecnalia.com \\ ${ }^{\mathrm{b}}$ Fundación Especial Caja Madrid, Madrid, Spain. ana.almagro@gmail.com \\ CIPA Heritage Documentation XXIV International Symposium
}

KEY WORDS: Technology education, training, capacity building, cultural heritage, conservation, preservation education, International Digital Divide

\begin{abstract}
:
Technology to document and investigate cultural heritage sites is rapidly advancing - multispectral and high dynamic range imaging, spherical high resolution photography, three-dimensional laser scanning and unmanned aerial vehicles are only a few of the new technologies available to heritage conservation professionals to record monuments, buildings, city centres and landscapes. These advanced tools are giving architects, engineers and conservation professionals' new insights and additional information which helps to make better informed decisions.
\end{abstract}

But this technology and the knowledge about its correct use are extremely unevenly distributed across the world. The Digital Divide is present and growing in the field of cultural heritage preservation (Letellier, 2001). Many of those responsible for the management, maintenance and care of some of the world's most significant cultural heritage sites do not have access to or information about the latest technologies. They are also confronted with an overwhelming assortment of new technologies and consultants or developers that promote them and therefore must allocate their limited budgets with limited information.

What is to be done about bridging this gap? Obviously cost and accessibility are issues. However one of the most important challenges to be addressed is education. As the base knowledge of these technologies is very uneven this leads to further questions: Are there strategies or methodologies for teaching this technology? How to combine and balance different professional backgrounds from different and so unevenly distributed places around the world and provide them all with useful information to make good documentation and conservation decisions?

This paper will describe the methodology developed over the last ten years in teaching documentation technologies to diverse groups of cultural heritage professionals and students from Côte d'Ivoire, Germany, Belgium, Kosovo, Albania, Nigeria, Egypt, Japan, Iraq, Jordan, Argentina, United Arab Emirates, United States of America and around 20 other countries. These strategies deal with establishing methodologies and guiding principles for the selection of technologies, highlighting successful illustrated examples, levelling uneven educational bases and gaining access to expertise. The authors have developed these strategies and techniques to appeal, engage and succeed with such diverse groups - to encourage the participants to cooperate on a common goal and overcome specific challenges while embracing the technology and thinking critically about its appropriate application for the conservation of cultural heritage in their home countries. Other strategies include setting norms that respect the various cultures and differing levels of technology education, offering voluntary sessions for more advanced and ambitious participants, finding and then adopting natural leaders as co-instructors and offering a mix of sessions including standard lectures combined with field and laboratory exercises and distance learning. This methodology and strategies have proven to be successful as the participants have provided positive evaluations months and/or years after the courses, implemented their own courses using the materials and methods and have established a strong, sustainable network related to this topic.

\section{INTRODUCTION}

A single monument, archaeological site, group of buildings, landscape or historic town centre are cultural places or locations that have a special meaning and values including physical, historical and spiritual aspects. The protection or conservation of such places means all the processes of looking after it as to retain its cultural significance. This involves all efforts, measures and actions to safeguard, protect, preserve, and transmit cultural significance (Australia ICOMOS, 1999).

This abbreviated definition includes an in-depth understanding of a cultural place in order to properly provide protection. This understanding is achieved, in part, through documentation or evidence (Clark, 2001). Documentation functions as both a verb and a noun and is a collection of data and/or information that assists in reaching informed conservation decisions. Increasingly the information needed to make these decisions is obtained through the use of technology and recording tools (Letellier-Leblanc, 2007). Some of these technologies have only recently been developed and older tools have undergone radical improvements. Reliance on technology for documentation has been on the steep upward slope of the adoption curve for at least the last fifteen years (Eppich-Hadzic, 2013) (Figure 1). 


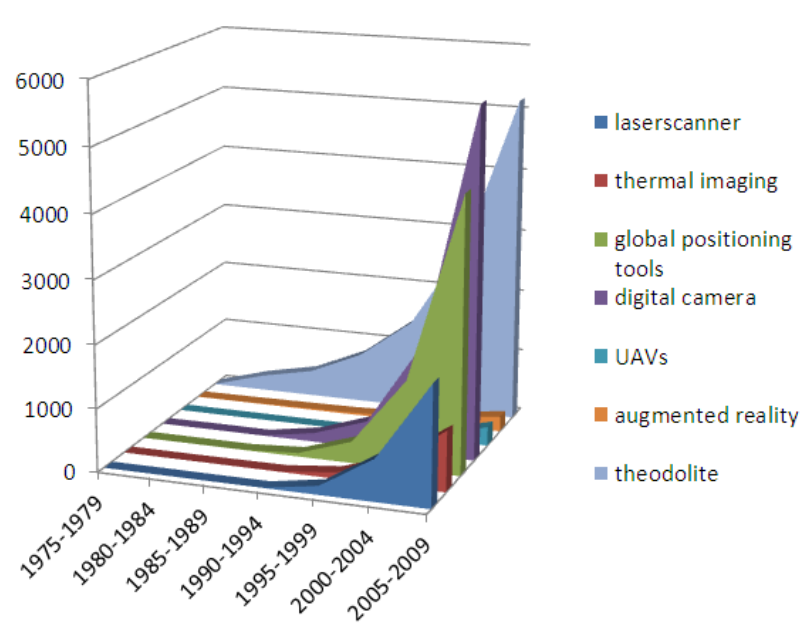

Figure 1. Number of articles published concerning advanced technology for documentation in five year segments. This article graph clearly shows an increase in the use technology

(Eppich-Hadzic, 2013)

\subsection{The Digital Divide}

This increase in the use of technology for documentation of cultural heritage places has led to a gap or divide between those who use it and those who do not have access, understanding or the required budgets. The International Digital Divide (Rogers, 2001) -access to technology- is well known around the world in many varied professions. Specifically within the conservation profession this divide is present and rapidly growing (ChenWellman, 2004). This divide is due to a unique combination of circumstances:

1) Multidisciplinary. The problems associated with the conservation of historic places are varied, complex and range from the materials and environmental situation to legal protection, economic and social values. Therefore the field requires a wide variety of talents in order to successfully protect a historic place. This results in a diversity of backgrounds and educations and thus differing levels of knowledge, education and comfort with technology.

2) International. Historic places are widely dispersed around the world with many places in the developing world where access, knowledge or resources to use the most recent technology are not readily available. This is simultaneously made better and worse as there are many international nongovernmental and inter-governmental agencies seeking to protect these places that do have the resources and thus highlight and emphasize this divide.

3) Funding. The conservation profession is financially challenged in comparison to other fields. Conservation works are usually time consuming due to the uniqueness of each place and understanding the associated problems, the necessity of in-depth historic and material research. In addition practitioners often require extensive and advanced training.
4) Attractive. Many historic places around the world such as the Taj Majal, the Alhambra or Notre Dame are all very attractive and famous places and are often used to help "selling" advanced technology. Inventors and developers of technology often like to point their devices/inventions/creations at these attractive famous sites rather than other heritage places that have other nonaesthetic values. They also like to be seen as doing "good" by helping to record these places. While this is of great service, this paradox provides exposure to the latest technology at the most attractive places while often ignoring other less well known or less attractive locations that are just as worthy of the application of the latest technology. In addition many of these famous sites are recorded multiple times, resulting in overlapping documentation projects.

5) Innovation. The pace of technology is accelerating. New developments in 3D laser scanning, multispectral imaging and UAV technology (Unmanned Aerial Vehicles), to name a few, are giving the field new choices every year. Even specialists or professional users have sometimes difficulties keeping up with the latest technology.

What is to be done about bridging the Digital Divide within cultural heritage conservation? Obviously cost and accessibility are issues. However the most important challenge is education.

Are there any strategies or methodologies for teaching this technology? How to combine and balance different professional backgrounds from different and uneven places around the world? Given the challenges outlined above specialized teaching techniques are necessary to close or bridge this gap especially when working with a diverse, international group of professionals with a wide variety of applications and technology. This methodology and these teaching techniques evolved according to this situation, but were also deliberately developed to address the specific challenges to cultural heritage conservation.

This paper is a summary of over 10 years of training, university and capacity building programs conducted under the aegis of ICCROM (International Centre for the Study of the Preservation and Restoration of Cultural Property) (Almagro Vidal, 2005), The Getty Conservation Institute (GCI), UNESCO World Heritage Centre, World Monuments Fund, Escuela de Estudios Árabes-CSIC, Fundación Caja Madrid and Tecnalia Research \& Innovation (ICCROM-GCI 2003, 2005, 2007, 2009). These programs have lasted from two months to just a few days and included participants from both sides of the International Digital Divide. This paper will describe the background and objectives of the courses, strategies developed for teaching technology, methodology and shortcomings. Results will also be described as well as conclusions.

\subsection{Methodology}

The following teaching methodology for technology was developed in several ways: first, in consultation with experienced international educators, experts and colleagues in the field from the various institutions sponsoring these programs as well as the authors' own experiences teaching and lecturing at universities. Advice was obtained from three fields: conservation, education and technology. The methodology also included incorporating extensive research and reading published materials on technology training. New documentation 
techniques were also incorporated into the methodology by trial and error, assessing results over the course of 10 years. This gave the authors a laboratory to experiment and refine teaching techniques. Finally, and most importantly, the participants and instructors have been extremely useful in providing honest direct feedback. They have been open and communicative immediately after the course but also years later and sometimes justifiably harsh. Both participants and instructors have gone on to form their own courses, have used the material and have provided additional feedback, which led to further refinement of the methodology. The course methodology will be described in three phases - pre-course, during the course and follow-up after the course.

\section{PRE-COURSE}

The first step in this methodology was extensive preparations before the courses, often many months and in some cases years before the courses began. This point cannot be stressed enough, that there was sufficient time to prepare the materials, coordinate the instructors and select and communicate with participants. This differs from many regular courses in technology as there was no set schedule as at a university or secondary education level. Many times the cultural response rate or frequency of communication is very different depending upon the customs of the participants' countries. In North America and Europe the expected response rate to an email or telephone call is within 24 hours but this is not the case in many other regions of the world, therefore sufficient time was required to expect responses and pre-course communication time was a critical issue.

\subsection{Preparation}

Selection: During course formation very careful selection was made of all course participants through a paper/on-line application. While this in itself is not unique the authors added a personal interview process to understand the motivations and needs of the potential participants. This process included specific questions formulated and presented to the applicants about their expectations, experience and course requirements adjusted for various levels of technology education. Extreme care was exercised as to not bias the results. The review process for participants was individual with a final discussion and weighed evaluation that included the needs and available resources of the participants' home countries.

During this phase gaps in the potential participants' technical knowledge were uncovered. This helps later in assigning prework to the participant to assist them in preparing for the course. This individual contact was important in determining the technical knowledge as well as English language capabilities. This careful selection phase helped the coordinators custom tailor the course to fit the group of participants. For example, one participant from Côte d'Ivoire required a country wide survey and stated this in the application, therefore the coordinators changed the preliminary program to include a global positioning technology exercise to reach his specific expectations, which resulted also very useful for many others. This proved to be one of the most successful course modules.

Short Duration: Because most international training courses are very short in duration it was important to maximize the time available. Therefore the participants that were finally selected were assigned tasks to accomplish before they arrived at the training locations. Such tasks included studying tools or methods that were identified during the application process. This would help them arrive prepared and maximize the time available before the course began. The course also had a preparation week that was less intensive. This allowed participants to arrive early and have one-on-one sessions if they required specific training before the course began. It also allowed the coordinators a 'buffer' to ensure that the main course began on time. This 'buffer' was necessary as many participants had visa issues and were coming from long distances and therefore with a need to recover from jet-lag.

\subsection{Establishing Norms}

All participants upon arriving to the training sessions had a oneon-one discussion with a course coordinator. This private meeting was essential as it allowed the more timid participants or those with limited English skills time and space to talk and understand the expectations of them as well as inform them of the course standards. These standards or norms included respect for other participants' culture, background and level of technological knowledge. This was extremely important as many participants arrived from countries or territories that are at ideological odds with one another such as Palestine and Israel, Former Yugoslavian Republic of Macedonia and Macedonia, Greece. It also conveyed the idea that not everyone is beginning from the same technological base and there must be tolerance and understanding between individuals; that the technological knowledge is not a factor in the value or worth of an individual. These one-on-one discussions also assisted in discovering special talents of the individuals that may not have been described during the application process, and to reinforce each participant to share their specific know how, to be open to help the others as far as possible. This establishing of norms helped in many aspects of the course including team forming and personal introductions, coordinator introductions and put the courses on a personal level of cooperation.

\section{COURSE WORK}

The pre-course preparations assisted greatly in preparing both the participants as well as the coordinators. It informed the coordinators about the technological level of the incoming group of participants and allowed fine-tuning of the course materials and schedule. Once the course began:

\subsection{Active Involvement}

Participants: Part of the course norms include that those involved in the training exercises are participants and not students. They were informed that they are required to actively participate, involve themselves not as a passive student but as engaged members of the programme. It was communicated that their active involvement was absolutely necessary for a successful course. With such small and short courses there was no room for passive 'listening'. An important part of this involvement included helping others with less of a technological base. For example, one of the software that is necessary for digital drawing and processing data in these courses is AutoCAD (or similar programs). Not all participants had sufficient knowledge of this technical software. Therefore those participants who had knowledge of this software were told that they must help others to using it; that they are in essence also instructors and coordinators. This resonated well 
with many of the participants as they took an active role in assisting other participants with less technical knowledge.

Presentations: Another part of their active involvement was that the participants were required to present their own specialized work - reinforcing the idea that they were also instructors. Time was made available for the participants to lecture to the others on their own work, use of technology and specific needs and problems of their countries. This accomplishes three things. First, it engages the more reluctant participants; secondly, it informs the other participants of the situation in other regions of the world; and third, it gave a sense of pride and recognition of the work of the participants and allowed them to speak out and prevented the discussions from being dominated by more vocal individuals. This was particularly important for those with less developed English or technological skills. Often the coordinators discovered that many of the participants have specialized technical work that in some cases exceeded that of the instructors.

Team Forming: One of the most successful aspects developed during these training courses was team formation. Carefully chosen teams comprising of 2-5 individuals were formed balancing in terms of technical abilities and knowledge, backgrounds and language skills. If one participant knew more about digital photography they were paired with an individual that did not know this subject. In the cases where these teams are larger they are often asked to elect a spokesperson. Many of these team members become lifelong friends maintaining contact years after the training courses.
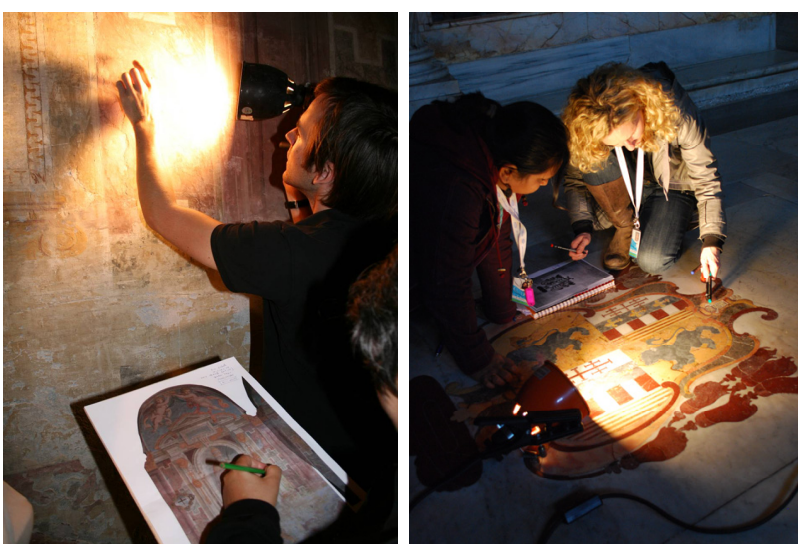

Figure 2 and 3. Left, team evaluating wall frescos at the ICCROM-GCI ARIS course, 2007

Right, team evaluating stone deterioration at the ICCROM Built Heritage Course, 2012

\subsection{Product Goals}

Final Product: One of the binding factors during these training sessions is that at the end of the course a final finished product was expected. This was done for several reasons; first, if there was a common goal often participants worked harder and cooperated together to accomplish this goal and secondly it provided needed information for an upcoming conservation project. The participants were informed of the clear intentions and outcomes of this final product and the need to accomplish this goal. For example, in the damaged frescos of the Church of Santa Cecilia in Rome the paintings were to undergo conservation treatment and the superintendent responsible for the works required a base record of the walls. The participants were informed of this work and it motivated them to complete a project and work together. After the course the results were presented to the superintendent and a small publication produced.

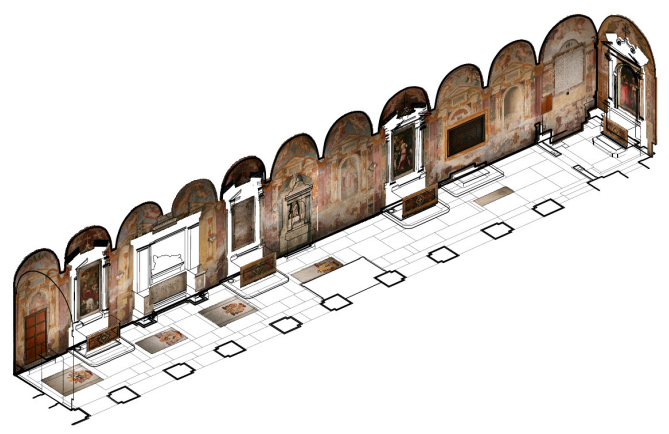

Axonometric Left Aisle,Santa Cecilia

$$
1: 1000 \quad 1
$$

Figure 4. Final product of the ICCROM-GCI training program in 2007. The 3D model with rectified photographs of the left aisle of the Church of Santa Cecilia, Rome

Connecting Years: During the courses developed in Rome a presentation of other past courses was always scheduled. This set the standard for their work and gave participants a goal for meeting or exceeding the work of past courses. It also informed them that their work would be appreciated in coming years and held in high regard. In addition a key past participant from the previous year was invited to give a lecture on how the course was helpful to their career. This created a link to past programs and also motivated participants to become that key participant for future courses. Building on other courses - the work and final product of these courses - often built upon the work of the previous courses. This allowed the participants to feel as if they are part of a larger movement and that their course was not an isolated "one-off" course. For example, in the case study mentioned earlier the participants used technology to create the documentation for the left aisle of Santa Cecilia. In the following years the right aisle was documented and then during another course the tombs on the floor were the focus. This has led to a greater "product" rather than the one that only a single course could have created.

\subsection{Innovations}

Balance: During these technical training sessions there was always a balance sought between lectures, field exercises and technology laboratory exercises. Any one of the previous would overtax an individual. This balance was carefully monitored during the on-going course evaluations and adjusted accordingly. This was also reflected in the schedule as there were built-in flexibilities in the schedule that allowed for the readjustment of this balance. Many of the instructors were asked to prepare their material to either be lectures or exercises. There was also additional time scheduled that was adjusted to a lecture overrun or discussion or demonstration. Several "floating" demonstrations and exercises were prepared in advance to maintain this balance. For example, an exercise on aerial balloon photography was prepared in advance and was ready for when the lecture/exercise/lab ratio was out of balance. 
When the coordinators felt this was the case an additional 20 minutes was set aside to carry out this exercise. This provided an element of surprise and generated enthusiasm during a particularly difficult or long series of lectures or lab processing work.

Social Activities: Technical training courses were observed to succeed most often when the participants were comfortable with each other and relaxed and able to openly ask questions to each other and to the coordinators or instructors. This was very difficult during short courses of only four weeks or less. Therefore planned social activities were an essential component of these courses. Not only was this an important component but when these activities occurred was also important. A social activity was always planned at the beginning of the course. This allowed the participants to get to know one another and the coordinators and first instructors in a social setting away from the stress of technical training sessions. This was a formal opening, but the participants were also encouraged to form their own social activities during the course. One such participant led social activity was an intercultural dinner where each participant brought a special "dish" from their own home countries. Finally a formal closing social session was held and official representatives from their home countries were invited to recognize their achievements.

Volunteer Sessions: Many of the participants arrived from one side of the Digital Divide where their technical knowledge base was much higher than others. With such a mixed international group if the course was directed toward those with more knowledge those from less fortunate countries would have been 'lost' and vice versa, if the course material is mainly directed toward less fortunate individuals then the participants from more advanced countries would quickly become bored. This was remedied in two ways. First, the participants were informed about their responsibility also to be instructors and share their knowledge; and secondly, volunteer sessions were conducted. These sessions were organized externally after hours, during the evenings or on the weekends in order to give the more advanced participants an opportunity to exercise their greater technical interests and skills. For example, during one training session a voluntary excursion was at night to allow the participants to learn about night photography and artificial lighting technologies. This gave an outlet for coordinators and provided the excuse that the advanced participants could not state the course was insufficiently advanced because they were given the opportunity to have more technically oriented sessions.

\section{POST COURSE}

\subsection{Evaluation}

Pre-Course Evaluation: Constant evaluations were conducted to ensure that course coordination was on track. The first evaluation was required before the participants arrived at the training location. This provided a base level information on what the participant knew, what they were interested in and what their expectations were for the course.

On-going Evaluations: Participants were also evaluated every Friday about the work of the week. This gave coordinators the opportunity to discuss the shortcomings of the week and suggestions by the participants over the weekend. Then adjustments were made and the participants were informed of the results during the following Monday morning. For example, during one course at the half way point of the training program many of the participants provided feedback that they were afraid that they were not getting to know their fellow participants sufficiently. The coordinators evaluated this and decided to host an additional social event in the form of a pizza party. This was well received and achieved in rebalancing the course. These surveys were originally conducted by paper survey but then the online survey tool SurveyMonkey was used. It was important that these surveys were very short with no more than 20 questions that could be completed in 10 minutes. It was attempted to have the same evaluation form for different courses to compare across courses but this proved to be too difficult as the courses and content were constantly changing and were specifically adapted to each group of participants as well as improved following past participants feedback.

Final Evaluation: The final evaluation after the course was exactly the same questions as the pre-evaluation before the course. This allowed one-to-one comparisons to questions and knowledge before the course began. This was very helpful to the coordinators as a tool for future funding and proving success. $100 \%$ of all participants evaluated the courses and clearly stated that their technological abilities had improved. This was not too surprising given that they were just finishing the course.

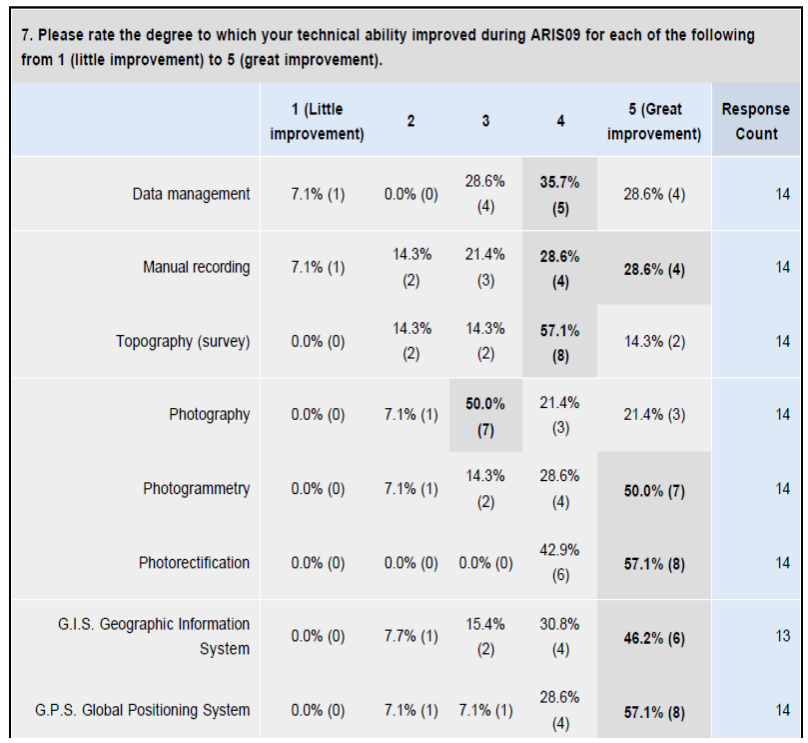

Figure 5. Evaluation sample from the ICCROM-GCI training course ARIS 2009

\subsection{Follow-up}

However the evaluations did not stop there, after one year the same evaluations were sent via email to the participants with an over $90 \%$ response rate. Even after one year the participants stated that the courses helped in improving their technical abilities and contributed toward improving documentation of cultural heritage places in their countries. Finally, an important aspect of the evaluation was the division between evaluating instructors, content and course coordination. This was extremely important because the coordinators wanted to understand if it was the content that was successful (or unsuccessful) or if the success was the instructor delivery or coordination. 


\section{CONCLUSIONS}

\subsection{Results}

In over 10 years and 28 training courses approximately 250 mid-career professionals were trained to use technology tools for the documentation of cultural heritage places around the world. These individuals included architects, planners, engineers, museum curators, conservators and other backgrounds. They came to the training courses with different levels of technical education but with the same goal of improving their knowledge of these tools in order to enhance conservation practice in their countries. Many of them had difficulty using basic graphic editing tools while, in the same course, there were participants attending who had advanced degrees and in some cases $\mathrm{PhD}$ with high levels of technical training.

Using the teaching methodology and techniques described earlier the goal was to improve technical ability and familiarity with new documentation tools in order to provide participants with a general overview of the field and some practice on the main tools available, so as to improve the conservation of cultural heritage places.

This paper is therefore the result of the development of a methodology to address this wide gap between technical education and the specific field of documentation for cultural heritage preservation. This methodology is still evolving and constantly being adapted as required by the subject itself. Technology is rapidly changing and the course materials and methods must also change and adapt. This is due to many new disruptive technologies such as the advancement of integrated devices, for example, the combination of GPS and cameras into smartphones or tablets. This often renders specific training useless unless these new options are taken into account, analysed and assessed for our specific documentation goals.

The authors and coordinators of these courses feel that almost all participants benefited from the course experience. This is evidenced by the continuing strong network with more than $90 \%$ of the participants still in contact after more than ten years, the evaluation feedback and through the continued sources of funding to carry out these courses.
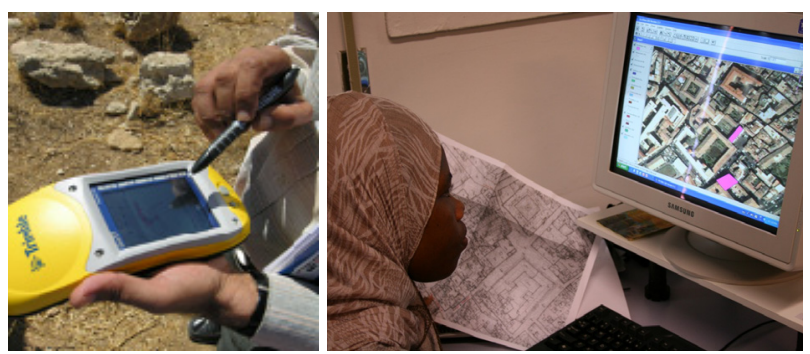

Figure 6 and 7. Training in the use of GIS during the GCIWMF training initiative in Jordan in 2005 (left) and during the ICCROM-GCI ARIS Course in 2007 (right)

\section{REFERENCES}

Almagro Vidal, A., 2005. Training on Documentation in Architectural Heritage: The Experience of ARIS and CLADIC Courses. CIPA XX International Symposium in Turin
Australia ICOMOS, 1999. The Burra Charter - the Australia ICOMOS Charter for Places of Cultural Significance

Chen, W., Wellman, B., 2004. The Global Digital Divide Within and Between Countries. IT \& Society, vol. 1, issue 7, spring/summer pp. 39-45

Clark, K., 2001. Informed Conservation, Understanding historic buildings and their landscapes for conservation. English Heritage

Eppich, R., Chabbi, A. (eds.), 2007. Illustrated Examples, Recording and Documentation for the Conservation of Heritage Places for Conservation, J. Paul Getty Trust, Donhead, 2011 second edition

Eppich, R., Hadzic, L., 2013. Heritage Conservation - Aligning Technologies, CICOP International Conference

Letellier, R., 2001. "Bridging the Gap" Between the Information User and the Information Provider. Working Group report CIPA Conference Potsdam

Letellier, R., 2004. Strategic Alliances as seen by ICOMOS and Information Users, ISPRS CIPA Special Session, Istanbul

Letellier, R., LeBlanc, F., 2007. Guiding Principles, Recording Documentation and Information Management for the Conservation of Heritage Places, J. Paul Getty Trust, Donhead, 2011 second edition

ICCROM, GCI Newsletters on the ARIS courses. Websites of institutions sponsoring training programs: www.iccrom.org, www.getty.edu, www.tecnalia.com, www.laac.csic.es

Rogers, E., 2001. The Digital Divide. Convergence, vol. 7 no. 4 pp. 96-111

Smith, J., 1978. The Scope of Heritage Conservation, Conservation Technology Manuals, Bulletin of the Association for Preservation Technology, Vol.10, No. 3, pp.33-38

\section{ACKNOWLEDGEMENTS}

The authors would like to thank and acknowledge the following institutions that made possible these training experiences over the last 10 years: ICCROM in Rome (Italy); ICCROM-ATHAR Regional Conservation Centre in Sharjah (UAE); Raymond Lemaire International Centre for Conservation, KU Leuven (Belgium); Tecnalia Research \& Innovation in the Basque Country (Spain); Escuela de Estudios Árabes-CSIC in Granada (Spain); The Getty Conservation Institute in Los Angeles (USA); World Monuments Fund, Cultural Heritage without Borders in Sarajevo (Bosnia and Herzegovina), as well as all the participants and their respective institutions that throughout these 10 years were trained during these courses. 